\title{
The Relative Significance of Factors Affecting Postoperative Survival in Astrocytomas, Grades One and Two
}

\author{
BRYCE WEIR AND MICHAEL GRACE
}

SUMMARY: The records of 107 patients with supratentorial astrocytomas, Grades $I$ and 2, treated surgically between 1960 and 1970 were analyzed. Abstracted clinical data was retrospectively analyzed with respect to the relative significance of different factors affecting survival. Twenty-eight patients were alive at the time of follow-up in 1974. Age, clinical grade at surgery and radiation therapy were the most important

RESUMÉ: Les rapports de 107 patients avec astrocytome supratentoriel, grades I et 2, traités par la chirurgie entre 1960 et 1970, furent analysés. Les données cliniques résumées furent analysées rétrospectivement en tenant compte de la signification relative des différents facteurs affectant la survie. Vingt-huit patients étaient vivants au moment du "follow-up" en 1974. L'âge, le degré clinique au moment de la chirurgie et la thérapie par radiation étaient les determinants of prolonged postoperative survival.

The more malignant astrocytomas occurring in the Province of Alberta had previously been reviewed. The present study was carried out to demonstrate the relative influence of histologic grade of astrocytomas and to analyze the factors affecting postoperative survival in the low grade, supratentorial astrocytomas.

causes de survies prolongées postopératoires déterminantes les plus importantes.

Les astrocytomes les plus malins survenus dans la province de l'Alberta ont été analysés antérieurement. La présente étude fut réalisée dans le but de démontrer l'influence relative du stade histologique des astrocytomes et d'analyser les facteurs affectant la survie post-opératoire chez les astrocytomes supratentoriaux peu évolués.
From the Dr. W. W. Cross Cancer Institute and University of Alberta, Edmonton, Alberta, Canada.

Reprint requests to Dr. Michael Grace, 11560 University Ave., Edmonton, Alberta T6G 1 Z2 Canada.

\section{CLINICAL MATERIAL}

In Alberta.between 1960 and 1970 there were 107 cases of Grade 1 and 2 supratentorial astrocytomas, along with 13 brain stem gliomas, 9 cerebellar astrocytomas and 3 astrocytomas occurring in the spinal cord. During the same time there were 248 supratentorial Grades 3 and 4 astrocytomas. By pathologic classification for all astrocytomas in Alberta, 1960-70, the percentages were Grade $1-8 \%, 2-24 \%, 3-$ $39 \%, 4-27 \%$, unspecified $-3 \%$; by location they were: cerebral $88 \%$, brain stem - 5\%, cerebellar $2 \%$, spinal cord $-0 \%$, unspecified - 4\% (to the nearest \%). Only the Grade 1 and 2 supratentorial astrocytomas were analyzed in detail.

The clinical information coded for computer analysis included the year of diagnosis, the surgeon, the patient's sex, age, duration of preoperative symptoms, postoperative survival in months, the presence or absence of headaches preoperatively, the estimated tumor size in centimeters based on the diagnostic studies, the type of diagnostic contrast study, the amount of midline shift in millimeters, the clinical group of the patient on admission preoperatively, the surgeon's estimate of the amount of tumor removal, the grade of pathology (1 or $2)$, steriod therapy (yes or no), and radiation therapy (yes or no).

\section{RESULTS OF ANALYSIS \\ General}

Nine neurosurgeons operated on 107 patients: $33 \%$ of patients had needle biopsy, $46 \%$ subtotal removals, $18 \%$ total removal, and $3 \%$ unknown. Radiation therapy was car- 
ried out in $71 \%$ of patients and omitted in $29 \%$. In $23 \%$ the astrocytomas were classified as Grade 1 and in $77 \%$ as Grade 2. Sixteen percent of the patients survived less than one month postoperatively. Fifty-eight percent survived longer than one year. In $32 \%$ of patients, the preoperative symptoms were less than one month in duration, whereas in $28 \%$ of patients they were longer than one year. At operation $23 \%$ of the patients had no neurologic deficit: clinical grade $0,64 \%$ had neurologic deficit: clinical grade 1 , and $13 \%$ had a neurological deficit plus depression of consciousness: clinical grade 2 . Twelve patients lived longer than 84 months (11\%) and five patients were lost to follow-up. Eleven patients on second operative procedures were diagnosed as having grades 3 or 4 astrocytoma on the second biopsy specimen. The average survival for astrocytomas, Grade 1, was $52 \pm 39$ months, for the Grade 2 astrocytomas it was $30 \pm 34$ months.

\section{Statistical Analysis}

A chi square analysis was carried out relating each of the measured variables. Strong associations ( $p$ $<.001$ ) existed between duration of postoperative survival and both radiation therapy and age. Table 1 lists significant associations ( $p$ $<.05$ ).

The average survival was 33 months, with a standard deviation of 36 months (Table 2). In the earlier study of grade 3 and 4 astrocytomas $(\mathrm{n}=248)$ the average survival time was 6.6 months having a standard deviation of 8.1 months (Weir, 1973).

Table 2 also documents the number of cases and postoperative survival time by clinical grades for irradiated and non-irradiated groups.

The relationship of the extent of removal to postoperative survival is given in Figure 1. It is interesting that the percent survival is greater for sub-total than total removal for the first 9 months, but following that, the percent is greater for total than sub-total, which in turn is greater than for needle biopsy. The additional analysis of the extent of re- moval curves against the use of radiation postoperatively (Figure 2) indicates that the use of radiation therapy is more important than the extent of surgical removal. However, these distinctions become relatively trivial after 3 years.

Figure 3 shows the percent of survival over the months of survival for each of the three clinical grades. For long term survivors (more than 60 months) the clinical grades tend to converge. A further breakdown of the curves in Figure 3 into those who did or did not receive radiation appears in Figure 4. For the long term survivors there was no significant difference between those radiated and not irradiated, except for Grade

\section{TABLE 1}

Statistical Significance of Association of Various Therapeutic and Clinical Factors $(p<.05)$

Variables Analyzed

Probability

Postoperative survival and radiation therapy $\ldots \ldots \ldots \ldots \ldots \ldots \ldots \ldots \ldots . \ldots \ldots$

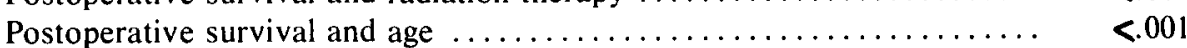

Postoperative survival and extent of removal $\ldots \ldots \ldots \ldots \ldots \ldots \ldots \ldots<. .05$

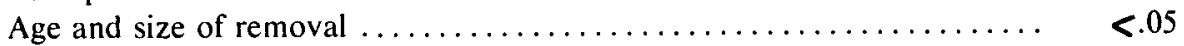

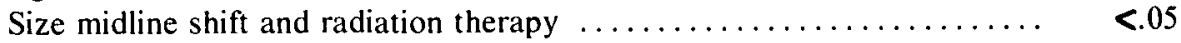

Clinical grade at surgery and steroids $\ldots \ldots \ldots \ldots \ldots \ldots \ldots \ldots \ldots \ldots \ldots \ldots$

TABLE 2

Postoperative survival in relation to clinical grade, radiation, and extent of tumor removal in 99 patients*

\begin{tabular}{lcccc}
$\begin{array}{l}\text { Clinical } \\
\text { Grade }\end{array}$ & $\begin{array}{c}\text { Irradiated Group } \\
\text { No. of } \\
\text { Cases }\end{array}$ & $\begin{array}{c}\text { Average } \\
\text { Survival (Mos.) }\end{array}$ & $\begin{array}{c}\text { Non-Irradiated Group } \\
\text { No. of } \\
\text { Cases }\end{array}$ & $\begin{array}{c}\text { Average } \\
\text { Survival (Mos.) }\end{array}$ \\
\hline Grade 0 & 18 & $50 \pm 32$ & 3 & $28 \pm 47$ \\
\hline $\begin{array}{l}\text { Needle Biopsy } \\
\text { Subtotal Removal }\end{array}$ & 1 & $37 \pm$ & 0 & $-\overline{1}$ \\
Total Removal & 6 & $52 \pm 37$ & 2 & $42 \pm 57$ \\
\hline Grade 1 & 44 & $29 \pm 30$ & 20 & $35 \pm 47$ \\
\hline Needle Biopsy & 18 & $25 \pm 33$ & 8 & $5 \pm 11$ \\
Subtotal Removal & 22 & $30 \pm 29$ & 7 & $50 \pm 49$ \\
Total Removal & 4 & $44 \pm 27$ & 5 & $63 \pm 60$ \\
\hline Grade 2 & 9 & $37 \pm 32$ & 5 & $<1$ \\
\hline Needle Biopsy & 3 & $30 \pm 40$ & 3 & $<1$ \\
Subtotal Removal & 5 & $43 \pm 34$ & 2 & $<1$ \\
Total Removal & 1 & $25 \pm$ & 0 & $<\overline{11}$ \\
TOTAL & 71 & $35 \pm 32$ & 28 & $28 \pm 44$ \\
\hline
\end{tabular}

* Mean survival in 99 patients was 33 mos. $( \pm 36)$ 
at surgery contributed $7.5 \%$, with the stepwise inclusion of other variables, such as radiation and extent of removal being lesser factors (explaining approximately $1.5 \%$ each of the variance not already explained by age and clinical grade) in predicting postoperative survival. Thus, only age and clinical grade at surgery were found to be significant in this regard. The resulting equation was: $y=81.7-0.91 x_{1}-10.4 x_{2}$

where $\mathrm{y}$ is the postoperative duration in months, $x_{1}$ is the age, and $x_{2}$ is clinical grade at surgery. The other variables and unknown factors beyond the two variables used would account for the remaining
$63 \%$ of the variance. It should be considered that only 50 cases were used in this analysis, just those having complete information on every variable reviewed.

In Figures 5 and 6 the postoperative duration of survival is plotted against age with and without radiation.

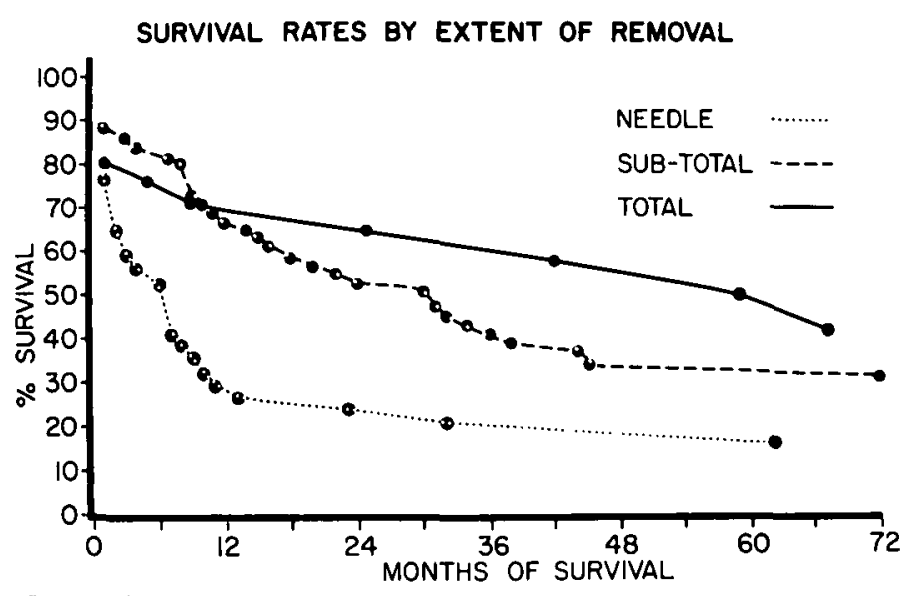

Figure 1

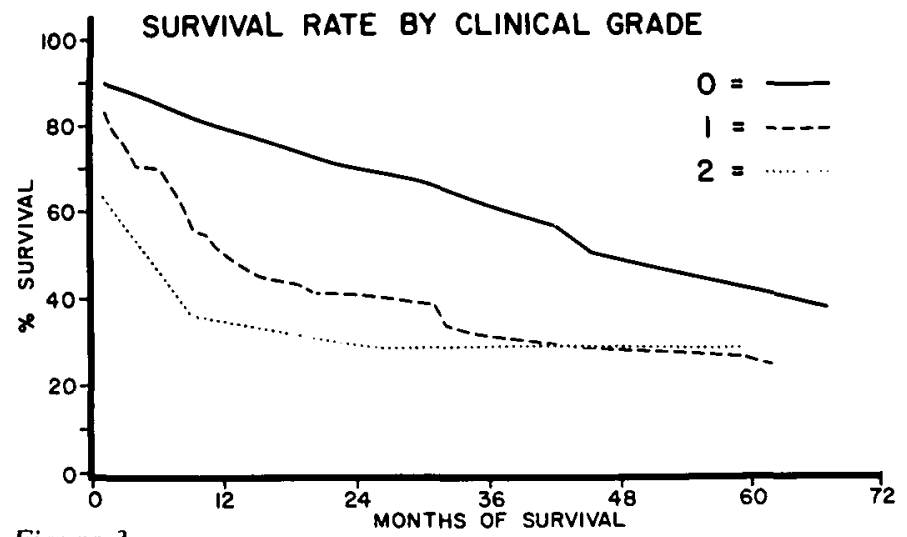

Figure 3

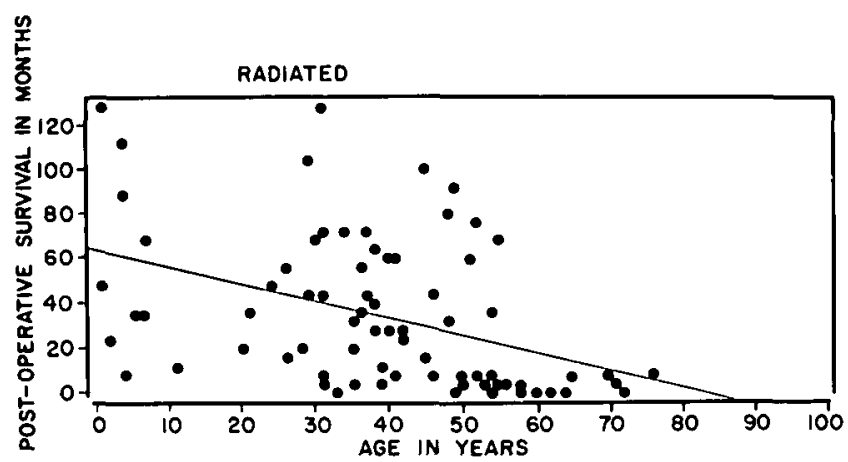

Figure 5

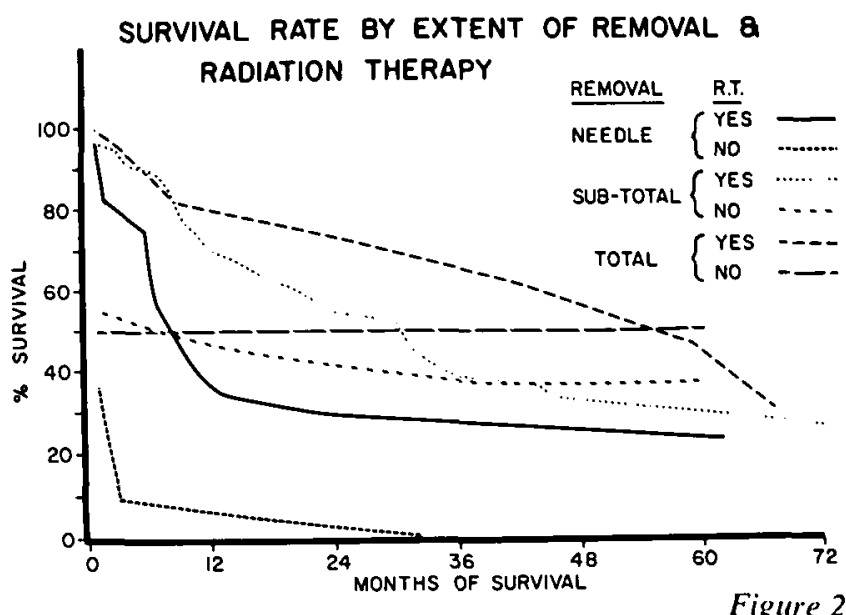

SURVIVAL RATE BY CLINICAL GRADE
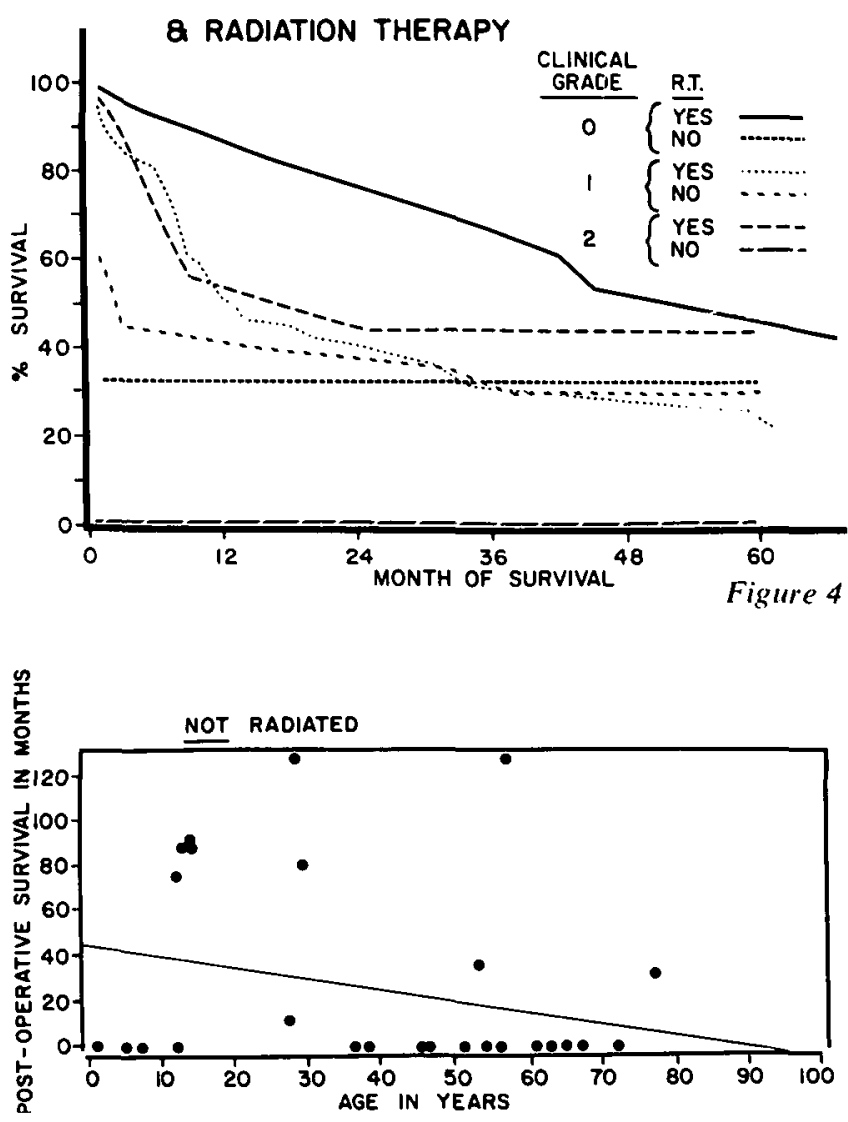

Figure 6 


\section{DISCUSSION}

This series is unique in that all cases in a given area and time were included. Postoperative deaths were not excluded and few cases were lost to follow-up. This would explain some of the discrepancies in survival data compared to other series of low grade astrocytomas (Cushing, 1932; Gol, 1961; Levy and Elvidge, 1956). There would also be a variation in standards of pathologic classification.

The $16 \%$ postoperative (1 month) mortality is dismaying considering the fact that these patients had low grade astrocytomas at surgery. This probably reflects the fact that some more malignant tumors were included because the surgical specimen consisted only of tissue of a lower histologic grade. These patients then might not have had autopsy and so remained classified as a lower grade astrocytoma. Despite the fact that these tumors were low grade, 14 of the patients came to surgery with some degree of impairment of consciousness probably reflecting a large intracranial mass. The mortality in this series, however, is comparable to that of the previous major series. Levy and Elvidge (1956) had a $13 \%$ postoperative mortality in 113 operated cerebral astrocytomas. Gol (1961) had a total case mortality of $27 \%$ in 194 histologically confirmed low grade astrocytomas; in that series the mortality for a resection was $16 \%$ and for a biopsy, 37\%. In Cushing's (1932) 164 cerebral astrocytomas, only 149 were operated and the case mortality was $15.4 \%$ of the operated cases. Ley et al. (1962) had a $16.2 \%$ postoperative mortality for cerebral astrocytomas. It is not possible to accurately compare these series because of the previous tendency to classify death occurring in hospital, regardless of time following surgery, as postoperative deaths. This is a less accurate method than specifying time. Factors other than the neurosurgical would come into play such as the ease of transferring patients to other facilities. With improvements in anesthesia, radiology and internal medicine current mortality can be expected to be reduced. - Weir (1975) had a zero postoperative (within 30 days) mortality in 27 Grade 1 and 2 cerebral astrocytomas.

The initial 4 grade classification system from the Mayo group, as an accurate prognostic system, appears to have significant limitations. In the present series, the difference between Grade 1 (52 months) and Grade 2 (30 months) was not statistically significant. Similarly, Gol (1961) found no marked difference in survival between grades 1 and 2 (106 cases). Weir (1973) found almost no difference in survival between Grades 3 and 4 (248 cases). On the other hand, the difference between Grades 1 and 2 (33 months) and Grades 3 and 4 ( 7 months) is very impressive. The Mayo classification divides Grades 1 and 2 from 3 and 4 by the presence of mitotic figures. From the point of view of estimating an individual patient's prognosis, it is probably most useful to consider whether he has a low grade (amitotic, 1 or 2) or high grade (mitotic, 3 or 4) tumor. One is always left with the important limitation that the surgical specimen may not be from the most malignant portion of the tumor.

It is apparent from this analysis that age is the most important indicator of postoperative duration of survival. Although clinical grade does not have a significant association with survival (Table 1) in conjunction with age, the stepwise regression shows clinical grade to be the most important predictor from all the remaining variables. This contrasts with the relative importance of radiation therapy that was found in a larger number of Grade 3 and 4 astrocytomas. In the lower grade astrocytomas, the extent of surgical removal appears to be relatively more important than in the higher grade tumors.
Gol (1961) found that "resection" was better than "biopsy", that young patients did exceptionally well and that radiated patients did slightly better than non-radiated patients.

In our patients who are still alive (4 - 14 year survivors) and presumably have some type of superior biological immunity to their tumors, it does not seem to be important whether or not they received radiation therapy or whether or not they had large removals carried out.

In lower grade astrocytomas it appears that a more aggressive surgical and radiotherapeutic approach is indicated than for the higher grades of pathologic tumors. The ultimate outcome for the patient will depend most importantly however, on host defences to this neoplasm, rather than any therapeutic activity on the part of his physician.

\section{ACKNOWLEDGEMENT}

We wish to acknowledge the invaluable assistance of Herta Gaedke in the collection and processing of data.

\section{REFERENCES}

CUSHING, H. (1932). Intracranial tumors. Notes upon a series of two thousand surgically verified cases with surgical mortality percentages pertaining thereto. Charles $C$. Thomas, Springfield, Illinois.

GOL, A. (1961). The Relatively Benign Astrocytomas of The Cerebrum. A clinical study of 194 verified cases. Journal of Neurosurgery, 18, 501-506.

LEVY, L. F. and ELVIDGE, A. R. (1956). Astrocytoma of The Brain and Spinal Cord. A review of 176 cases, 1940-1949. Journal of Neurosurgery, 13, 413-443.

LEY, A., LEY, A. JR., GUITART, J. M. and OLIVERAS, C. (1962). Surgical management of intracranial gliomas. Journal of Neurosurgery, 19, 365-374.

SVEIN, H. J., MABON, R. F, KERNOHAN, J. W. et al. (1949). Astrocytomas. Proceedings of the Mayo Clinic, 24, 54-64.

WEIR, B. (1973). The Relative Significance of Factors Affecting Postoperative Survival in Astrocytomas, Grades 3 and 4. Journal of Neurosurgery, 38, 448-452.

WEIR, B. (1975). unpublished data. 